130

Consistent Efficacy of DR/ER-MPH on Early Morning Functioning in Children With ADHD: Analysis of BSFQ Item Ratings From a Pivotal Phase 3 Trial

Timothy E. Wilens, $M D^{\prime}$; Steven R. Pliszka, MD'; Valerie K. Arnold, $\mathrm{MD}^{3}$; Andrea Marraffino, $\mathrm{Ph} \mathrm{D}^{4}$; Norberto J. DeSousa, $M A^{5}$; Bev Incledon, PhD ${ }^{5}$; F. Randy Sallee, $M D, P h D^{5}$; and Jeffrey H. Newcorn, $M D^{6}$

${ }^{1}$ Massachusetts General Hospital, Boston, Massachusetts, USA

2 The University of Texas Health Science Center at San Antonio, San Antonio, Texas, USA

${ }^{3}$ University of Tennessee Health Science Center, Memphis, Tennessee, USA

${ }^{4}$ Meridien Research, Inc., Maitland, Florida, USA

${ }^{5}$ Ironshore Pharmaceuticals \& Development, Inc., Camana Bay, Grand Cayman, Cayman Islands

${ }^{6}$ Mount Sinai Medical Center, New York, New York, USA; Onbehalf of the HLD200-108 Study Group

ABSTRACT: Objective: In a phase 3 trial of children with attention-deficit/hyperactivity disorder (ADHD), DR/ERMPH (formerly HLD200), a delayed-release and extendedrelease methylphenidate, improved ADHD symptoms and reduced at-home early morning and late afternoon/ evening functional impairment versus placebo. The validated Before School Functioning Questionnaire (BSFQ), a key secondary endpoint, was used to measure early morning functional (EMF) impairment. This post hoc analysis evaluated the effect of DR/ER-MPH versus placebo on individual BSFQ item scores from baseline.

METHOD: Data were analyzed from a pivotal, randomized, double-blind, multicenter, placebo-controlled, parallelgroup, phase 3 trial of DR/ER-MPH in children (6-12 years) withADHD (NCT02520388). Using the 20-item BSFQ, investigators evaluated EMF impairment by scoring each item on a severity scale of 0 to 3 , with 0 denoting "no impairment" and 3 denoting "severe impairment". For post hoc analyses, treatment comparisons between DR/ER-MPH and placebo at endpoint were determined by using least squares mean changes from baseline on individual BSFQ items score derived from an analysis of covariance (ANCOVA) model with treatment as the main effect, and study center and baseline score as covariates.

RESULTS: Of 163 children enrolled across 22 sites, 161 were included in the intent-to-treat population (DR/ER-MPH, $\mathrm{n}=81$; placebo, $\mathrm{n}=80$ ) and 138 completed the study. The mean DR/ER-MPH dose achieved after 3 weeks of treatment was $68.1 \mathrm{mg}$. Following 3 weeks of treatment, DR/ER-MPH significantly reduced mean BSFQ item scores frombaseline on 18 out of 20 items versus placebo $(\mathrm{P}<0.001$ in 8 items [listening, following directions, attention, forgetfulness, talkativeness, silliness, time awareness, getting to school]; $\mathrm{P}<0.01$ in 7 items [overall organization, being quiet, distraction, interrupt/blurt out, breakfast, hygiene, independence]; $\mathrm{P}<0.05$ in 3 items [procrastination, hyperactivity, awaiting turn]). Only "dressing" and "misplacing/losing items" showed no significant between-group differences $(\mathrm{P}=0.171$ and $\mathrm{P}=0.175$, respectively). Distributions of the severity ratings for each item will be presented. No serious TEAEs were reported; TEAEs were consistent with methylphenidate.

CONCLUSIONS: Post hoc analyses revealed that DR/ERMPH significantly reduced 18 out of 20 individual BSFQ item scores versus placebo in children with ADHD. These findings are worth further exploration.

FUNDING ACKNOWLEDGEMENTS: Ironshore Pharmaceuticals \& Development, Inc.

132

\section{Effects of Valbenazine on Depression and Suicidality in Adults With Tardive Dyskinesia: Pooled Results of 3 Double-Blind, Placebo- Controlled Trials}

Gary Remington, MD'; Dao Thai-Cuarto, PharmD'; Joshua Burke, $\mathrm{MS}^{3}$; Scott Siegert, PharmD ${ }^{4}$; and Grace S. Liang, $M D^{5}$

${ }^{1}$ Senior Scientist in the Campbell Family Mental Health Research Institute and Chief of the Schizophrenia Division at the Centre for Addiction and Mental Health, Toronto, Ontario, Canada

${ }^{2}$ Director of Clinical Drug Safety, Neurocrine

Biosciences, Inc., San Diego, CA

${ }^{3}$ Director, Biostatistics and Data Management,

Neurocrine Biosciences, Inc., San Diego, CA

${ }^{4}$ Executive Director, Head ofMedical Affairs,

Neurocrine Biosciences, Inc., San Diego, CA

${ }^{5}$ Medical Director, Clinical, Neurocrine Biosciences, Inc., San Diego, CA

ABSTRACT: Study Objectives: Valbenazine (INGREZZA; VBZ) is a novel and highly selective vesicular monoamine transporter 2 (VMAT2) inhibitor that is approved for the treatment of tardive dyskinesia (TD) in adults. The randomized, double-blind, placebo (PBO)-controlled trials of VBZ evaluated the treatment of TD in patients with a primary psychiatric diagnosis (schizophrenia/ schizoaffective disorder or mood disorder) while on concomitant psychiatric medications to manage these disorders. Since treatment-emergent depression and suicidal ideation/behavior are important clinical concerns in psychiatric patient populations, data from these trials were analyzed to assess the effectsof once-daily VBZ on depression and suicidality. 
METHODS: Data were pooled from three 6-week trials: KINECT (NCT01688037), KINECT 2 (NCT01733121), KINECT 3 (NCT02274558). Outcome data were analyzed in the safety population by pooled VBZ doses $(40 \mathrm{mg}$, $80 \mathrm{mg}$ ) and PBO. Outcomes of interest included: treatment-emergent adverse events (TEAEs) related to depression or suicidality; mean score change from baseline to Week 6 in the Calgary Depression Scale for Schizophrenia (CDSS, for participants with schizophrenia/schizoaffective disorder) or the Montgomery-Åsberg Depression Rating Scale (MADRS, for participants with mood disorder); and, worsening from baseline in Columbia-Suicide Severity Rating Scale (C-SSRS) suicidal ideation scores. All outcomes were analyzed descriptively.

RESULTS: There were 400 total participants in the pooled safety population; 286 participants had schizophrenia/ schizoaffective disorder $(40 \mathrm{mg}, \mathrm{n}=82 ; 80 \mathrm{mg}, \mathrm{n}=70$; $\mathrm{PBO}, \mathrm{n}=134)$ and $114 \mathrm{had}$ a mood disorder $(40 \mathrm{mg}$, $\mathrm{n}=28 ; 80 \mathrm{mg}, \mathrm{n}=42 ;$ PBO, $\mathrm{n}=44$ ). Over one-third of participants had a lifetime history of suicidal ideation or behavior (40 mg, 45\%; $80 \mathrm{mg}$, 39\%; PBO, 37\%). Few participants had a depression- or suicide-related TEAE, with no apparent differences between VBZ and PBO: suicidal ideation (40 mg, 3.6\%; $80 \mathrm{mg}, 0.9 \%$; PBO, 2.2\%); depression (40 mg, 0\%; $80 \mathrm{mg}, 1.8 \%$; PBO, 1.1\%); depressive symptom (40 mg, 0.9\%; $80 \mathrm{mg}, 0 \%$; $\mathrm{PBO}$, $0.6 \%$ ); suicide attempt $(40 \mathrm{mg}, 0 \% ; 80 \mathrm{mg}, 0.9 \%$; $\mathrm{PBO}$, $0 \%$ ). Mean changes from baseline to Week 6 in depression scale scores were generally small and similar across treatment groups: CDSS total score $(40 \mathrm{mg},-0.5 ; 80 \mathrm{mg}$, -0.6 ; PBO, -0.3); MADRS total score $(40 \mathrm{mg},-0.2 ; 80 \mathrm{mg}$, -1.7; PBO, 0.6). Few participants had a shift from no suicidal ideation at baseline $(\mathrm{C}-\mathrm{SSRS}$ score $=0)$ to any suicidal ideation during treatment (C-SSRS score $=1-5$ ): $40 \mathrm{mg}, 3.9 \%$ (4/103); $80 \mathrm{mg}, 0.9 \%$ (1/111); PBO, $2.9 \%$ (5/174).

CONCLUSION: Data from 3 double-blind, placebocontrolled trials indicate that once-daily VBZ treatment was not associated with a worsening in depressionrelated symptoms or an increased risk of suicidal ideation or behavior.

FUNDING ACKNOWLEDGEMENTS: This study was funded by Neurocrine Biosciences, Inc.

\section{3}

\section{Cognitive Impairment Following Overdose on Lamotrigine}

Geetha Chandrashekar, $M D, P G Y-3^{\prime}$; David Ash, MD, $P G Y-3^{\prime}$; and Garima Singh, $M D^{2}$

${ }^{1}$ Psychiatry Resident, University of Missouri-Columbia, Columbia, MO
${ }^{2}$ Assistant Professor, Department of Psychiatry, University of Missouri-Columbia, Columbia, MO

ABSTRACT: Objective: To generate hypotheses, accumulate scientific data about rare presentations, and serve as a major educational tool.

METHOD: This is a retrospective case report of a patient in inpatient unit.

INTRODUCTION: Lamotrigine is a mood stabilizer with unique mechanisms of action. At therapeutic levels, it has been reported that Lamotrigine is neuroprotective and improves cognition. In this case, we present a patient who suffered significant cognitive slowing following overdose on Lamotrigine.

CASE: A 17yo white male with a diagnosis of Autism spectrum disorder and Bipolar disorder type 1 was admitted for bizarre behavior and profound cognitive impairment. His past psychiatric history was significant for two suicide attempts - first by overdose on baby aspirin and second by overdose on Lamotrigine, both of which had occurred about six months prior to his presentation and had each required an inpatient hospitalization. His family reported that since his overdose on Lamotrigine, he had been withdrawn, aloof, and appeared depressed. His school teachers had noticed significant decline in his memory, attention and concentration, and there had been noticeable impairment in his ability to follow commands or complete a task. On assessment, he was noted to have significant psychomotor slowing, latency in speech and thought blocking. At the time of his presentation, he was on Lithium $300 \mathrm{mg}$ BID. A careful review of his previous medical records revealed that he had been on a combination of Seroquel, Lamotrigine and Lithium prior to his overdose attempt on Lamotrigine. During this hospitalization, Seroquel was restarted. Patient tolerated the medication well. There were no safety concerns and he was deemed safe to discharge under $24 \mathrm{hr}$ supervision of his family. He has since been followed up in clinic. Although he continues to have some cognitive slowing, overall, he has demonstrated slow but steady improvement.

DISCUSSION: Lamotrigine acts by blocking voltage sensitive sodium channels. It also reduces release of glutamate, a major excitatory neurotransmitter in the central nervous system. Glutamate modulates synaptic plasticity, a property thought to be vital for memory and learning. While too much glutamate causes over activation of NMDA receptors resulting in increased intracellular oxidative stress and eventually apoptosis, too little glutamate may lead to decreased glutamate mediated postsynaptic excitation of neural cells and thus impacting memory formation, learning and cognition. At 